\title{
Tracing electron density changes in langbeinite under pressure
}

\author{
R. Gajda ${ }^{1}$, D. Zhang ${ }^{2}$, J. Parafiniuk ${ }^{3}$, P. Dera ${ }^{4}$, K. Woźniak ${ }^{1}$ \\ ${ }^{1}$ Biological and Chemical Research Centre, Department of Chemistry, University of Warsaw, \\ 101 Żwirki i Wigury, Warszawa, 02-089, Poland, \\ ${ }^{2}$ APS, University of Chicago, 9700 S Cass Ave, Bldg 434A, Argonne, IL 60439, USA, \\ ${ }^{3}$ Institute of Geochemistry, Mineralogy and Petrology, Department of Geology, University of Warsaw, \\ Żwirki i Wigury 93, Warszawa 02-089, Poland, \\ ${ }^{4}$ Hawaii Institute of Geophysics and Planetology, School of Ocean and Earth Science and Technology, University of Hawaii at \\ Manoa, 1680 East West Road, Honolulu, Hawaii 96822, USA.
}

romanbg@chem.uw.edu.pl

Detailed studies of electron density changes in a mineral called langbeinite $\mathrm{K}_{2} \mathrm{Mg}_{2}\left(\mathrm{SO}_{4}\right)_{3}$ under pressure have been performed. Single crystal X-ray data for this mineral under pressure (1GPa) were collected at the 13-BM-C beamline at the Advanced Photo Source (Argonne National Laboratory, USA). Additionally, complementary experiments at ambient conditions were performed on an inhouse diffractometers. Experimental results were complemented by theoretical calculations within the pressure range up to $40 \mathrm{GPa}$.

From the point of view of mineralogical processes taking part in the Earth mantle (and the mantles of other even extraterrestrial planets), establishing detailed changes of electron density in minerals under pressure is absolutely crucial to understand the nature and mechanisms of mineralogical processes. Combining both experimental charge density studies and high-pressure investigations is still a real challenge. This work is our continuation of our previous feasibility studies on experimental quantitative electron density investigations of electron density in grossular under 1GPa pressure [1].

Answering the questions how electron density distribution in langbeinite is affected by increasing pressure is obviously the main topic of this work. However, there are also some other issues which we would like to address. Are there any differences between experimental and theoretical charge density distributions obtained based on experimental data and theoretical dynamic structure factors? Are there any significant differences in properties of charge density distributions obtained for complete and incomplete high resolution X-ray diffraction data sets? Are there any differences in charge density distributions obtained for X-ray data collected with two different wavelengths of X-ray radiation? Should the data be absolutely complete to obtain reasonable experimental charge density distribution? When experimental data are impossible to be collected, is it reasonable to use theoretically calculated dynamic structure factors instead and refine theoretical models of electron density?

Langbeinite crystalizes in the cubic $P 2{ }_{1} 3$ space group. Its structure is composed of $\mathrm{SO}_{4}$ tetrahedra and $\mathrm{MgO}_{6}$ octahedra. Potassium cations which are placed in the voids between these polyhedra are surrounded by oxygen anions. Unfortunately, due to significant deformation, one cannot say that $\mathrm{KO}_{12}$ is a regular icosahedron. Although mentioned polyhedra seem to completely fill in the space, this schematic way of presentation is not the best one when topology of electron density distribution must be described.

Investigating changes of electron density as a function of pressure, we are going to compare electron density properties at BCPs, integrated atomic basins, changes of thermal ellipsoids. Obviously, raising pressure will cause shrinking of the unit cell and consequently, changes of electron density distribution. However, the question is how exactly such changes will manifest.

No doubt that polyhedra commonly used in mineralogy and crystallography are not useful representation of electron density as they neither have full representation of electron density of the central ion nor any of the corner's ions. So, from time to time returns an old question: how big atoms in crystals are [2]. Here we will answer this question and the other ones already mentioned above at the level of quantitative electron density distributions in our model mineral.

[1] Gajda, R., Stachowicz, M., Makal, A., Sutuła, S., Parafiniuk, J., Fertey, P. \& Woźniak, K. (2020). IUCrJ. 7, $383-392$.

[2] Brown, I. D. (2017). Struct. Chem. 28, 1377-1387.

Keywords: high pressure; electron density; theoretical structure factors 